\title{
REAL-TIME SPEECH RECOGNITION SYSTEMS
}

\author{
Hy Murveit \\ SRI International
}

\section{OBJECTIVES}

SRI is developing the hardware, software, and algorithms necessary to achieve real-time speechrecognition and spoken-language systems. As the first phase of this project, SRI is currently completing the design of a prototype that will be able to recognize from 3,000 to 9,000 words of continuous speech using bigram language models. This prototype is based on four specialpurpose integrated circuits. SRI is also continuing to improve the SRI DECIPHER speech recognition system, and enabling it to be incorporated in to SRI's architecture for the integration of speech recognition and natural language processing.

\section{RECENT ACCOMPLISHMENTS}

- Specified and designed (in cooperation with U.C. Berkeley) four special-purpose integrated circuits that will serve as the basis of our real-time large-vocabulary speech-recognition system.

- Designed computer cards for the implementation of the prototype hardware.

- Implemented software to run the the DECIPHER signal processing routines in real time on a TMS32025-based board designed at U.C. Berkeley.

- Improved the design of the DECIPHER speech recognition system, which achieves high accuracy speaker independent or dependent continuous speech recognition. This included developing techniques for automatically generating pronunciation networks from standard training corpora. These networks improved speaker-dependent and speaker-independent speech recognition accuracy.

- Developed search strategies for DECIPHER that allow it to be efficiently used in SRI's spoken-language system framework.

\section{PLANS}

- Complete the prototype system and build three working real-time recognition systems. These systems will be evaluated with an application that uses a statistical language model.

- Develop concrete specifications for real-time spoken-language systems. This will likely include modifications to the prototype system to allow it to work in SRI's spoken language system framework--primarily by adding hypothesis pruning to the prototype.

- Continue improving the DECIPHER system including the addition of consistency modeling to the system, and real-time implementations of noise-processing algorithms developed at SRI. 